\title{
LOCALLY WEAKLY FLAT SPACES ${ }^{1}$
}

\author{
BY O. G. HARROLD, JR. AND C. L. SEEBECK, III
}

Communicated by O. G. Harrold, December 12, 1966

We give an alternative form of a definition in [3] and outline a solution of the problem of placing strictly local conditions on an embedding of a $k$-manifold in an $n$-manifold that imply a locally tame embedding. Local properties generally may be divided into mutually exclusive classes, the medial and the strictly local. Examples of the first variety are local compactness, local flatness, etc. Examples of the second variety are local connectedness, local weakly flatness (see below).

Modulo the conjecture that a $k$-sphere in $E^{n}, n-k \neq 2, n>3$, cannot fail to be locally flat at precisely two points, we prove that the strictly local property local weakly flatness implies local flatness. Cantrell and Lacher [1] have given a proof of this conjecture when $n-m \geqq 3$ and Chernavskii [2] has announced a proof of the conjecture when $n-m \neq 2$ and $n \geqq 5$. However complete proofs of his results have not been made available as of this writing. An interesting corollary to our proof, which is independent of the conjecture above, is that an $m$-manifold $M$ in $E^{n}, n>3$, cannot fail to be locally flat at a Cantor set in bd $M$ which is tame in both bd $M$ and $E^{n}$.

Definition 1. Let $p$ be a point of the closed manifold $M^{k}$ in $E^{n}$ and $\epsilon>0$. Suppose there are positive numbers $\eta, \delta, 0<\eta<\delta<\epsilon$ and a family of collared balls $\left\{B_{t}\right\}$ whose interiors contain $p$, of diameter less that $\epsilon$, such that for

$$
\delta-\eta \leqq t \leqq \delta+\eta, \quad \dot{B}_{t} \cap M^{k}=\Omega_{t}^{k-1}
$$

is a sphere that has a tubular neighborhood

$$
\left(E^{n}, \Omega_{t}^{k-1} \times I^{n-k+1}\right) \approx\left(E^{n}, S^{k-1} \times I^{n-k+1}\right),
$$

where $I=[-1,1]$. It is assumed $\delta-\eta \leqq t \leqq \delta+\eta$ is identified with $I$ so that

$$
\Omega_{\delta}^{k-1} \times(u, 0, \cdots, 0) \subset M^{k}: \Omega_{\delta}^{k-1} \times(0) \times I^{n-k} \subset B_{\delta} .
$$

We say that $\Omega_{\delta}^{k-1}=\Omega_{\delta}^{k-1} \times(0, \cdots, 0)$ is consistent relative to $M^{k}$ and $\dot{B}_{\delta}$, or, that $\dot{B}_{\delta}$ meets $M^{k}$ nicely.

If these conditions hold for a sequence of positive numbers $\epsilon_{1}$, $\epsilon_{2}, \cdots$ converging to zero, $M^{k}$ is called locally weakly flat (abbreviated LWF) at $p$.

\footnotetext{
${ }^{1}$ Research supported by NSF-GP 5458.
} 
Let $(B, D)$ be a ball pair of type $(n, m)$, i.e. $B$ and $D$ are, respectively an $n$-ball and an $m$-ball, such that $D \subset B$ and $D \cap B d B=\operatorname{Bd} D$. We say $(B, D)$ is nice on its boundary iff there exists a homeomorphism

$$
H:(B, D) \times[0,1] \rightarrow(B, D)
$$

such that $\dot{B} \cap D$ has a tubular neighborhood in $\dot{B}$ and

$$
H(x, 0)=x \quad \text { for all } x \text { in } B .
$$

Definition 2. Let $M$ be an $m$-manifold in $E^{n}$. Call $M$ locally weakly flat at $x \in \operatorname{Int} M$ iff for each $\epsilon>0$ there exists an $n$-ball $B C E^{n}$ such that

(1) $x \in \operatorname{Int} B$,

(2) $\operatorname{diam} B<\epsilon$,

(3) $(B, B \cap M)$ is a ball pair of type $(n, m)$ which is nice on its boundary.

It is not difficult to show that these two definitions of LWF are equivalent.

Theorem. Suppose $M$ is a closed m-manifold, $N$ is an n-manifold, $n-m \neq 2, n>3 M \subset$ int $N$, and $M$ is LWF. In case $n-m=1$ suppose either that there is an n-manifold $N^{\prime} \subset N$ such that $M \subset b d N^{\prime}$ or that an $m$-sphere in the n-sphere cannot fail to be locally flat at precisely two points. Then $M$ is locally flat.

CoRollary. If $M^{k}$ is a $k$-dimensional topological sphere in the $n$ sphere, then $M^{k}$ is flat, i.e. unknotted.

First it is shown that the subset $Y$ of the manifold $M$ where $M$ fails to be locally flat (=LF) is a subset of a Cantor set. Next, it is shown that $Y$ is in a certain strong sense "tame." We call a Cantor set $Z$ tame in a manifold $N$ iff there is a tame arc $A$ containing $Z$. In fact, if $M \subset E^{n}$, then $Y$ is tame relative to both $M$ and $E^{n}$. This being so we can form a decomposition space of $E^{n}$ by shrinking certain $\operatorname{arcs} A_{1}, \cdots, A_{\lambda}$ to a point. The image $M^{\prime}$ of $M$ turns out to be homeomorphic to $M$ but imbedded in the quotient space $E^{\prime n}$ in such a way as to be LF except possibly at a finite collection of points.

By a Cantorian construction, a neighborhood of a point of $Y$ is mapped onto a standard ball so that the relative neighborhood of the point on the manifold is carried onto a subball. 
For $k=n-2, k=1, n=3$ the corresponding problem was solved in [4]. It is interesting to note that both a strictly local and a medial property were used in that solution.

\section{REFERENCES}

1. J. C. Cantrell and R. C. Lacher, Some conditions for manifolds to be locally flat (to appear).

2. A. V. Chernavskii, Isotopies in Euclidean spaces, Uspehi Mat. Nauk (6) 19 (1964), 63.

3. O. G. Harrold, A new local property of embeddings, Bull. Amer. Math. Soc. 71 (1965), 882-885.

4. O. G. Harrold, H. C. Griffith, and E. E. Posey, A characterization of tame curves in three-space, Trans. Amer. Math. Soc. 79 (1955), 12-34.

Florida State University 